\title{
Application of 3D Printing Technology in the Treatment of Distal Femur Bone Tumor
}

\author{
Rui Jiang ${ }^{1}$, Ziyan Zhang ${ }^{2}$, Han $\mathrm{Wu}^{1}$, and Chao Zhang ${ }^{3}$ \\ ${ }^{1}$ Department of orthopedics, China-Japan Union Hospital of Jilin University, Jilin 130033, China \\ ${ }^{2}$ Department of orthopedics, The Second Hospital of Jilin University, Jilin 130041, China \\ ${ }^{3}$ Department of ophthalmology, The Second Hospital of Jilin University, Jilin 130041, China \\ Corresponding Author: Chao Zhang: zhangchao0701@163.com
}

Keywords: 3D printing technology; bone tumor; rapid prototyping technology; individual digital plate

Abstract: Objective: To investigate the application of individual digital resection plate in the treatment of bone tumor. Methods: Twenty-three patients with bone tumor by spiral CT scanner and MRI. The data was reconstructed by 3D imaging reconstruction workstation. The operation was simulated and the individual resection plate was designed on the virtual 3D model according to the surgical procedure. With the rapid prototyping technology, the individual resection plate was produced to guide the reduction of the bone tumor. Results: The average follow-up times were 48 months. No local recurrence, endoprostheses infection, loosening and fracture happened. Average scoring was 21 points according to Enneking system. Conclusion: Individual digital reduction plate is helpful in accurate reduction of bone tumor and can also save the operation time.

Bone tumor is a common clinical disease, and its incidence is around 0.01. Bone tumor may happen at any position, but it often occurs around knee joint, around 50\%, so it severely affects the extremity function of patients. According to statistics, internal prosthesis replacement for large bone defects after tumor resection may cause 5-35\% infection. Additionally, loosening happens to tumor joint prosthesis much more often than common joint replacement. If occurred, these complications may lead to disastrous consequences, such as serious financial impairment of extremities, worsening pain, poorer quality of life, and much higher medical cost. In their studies, some scholars point out that inaccurate marginal resection of bone tumor may easily cause recurrence [2]. Bone tumor is volumetrically large, and invades extensively, so the dissection position is complicated, and it is very difficult to perform the surgery due to high technical requirements and numerous postoperative complications. For this reason, a new approach is proposed by utilizing computer aided individual osteotomy plate and 3D printing technology aided bone tumor resection, so as to realize accurate tumor resection, and it achieves satisfying clinical results. 


\section{Data and Methods}

\subsection{General Data.}

Twenty-three patients with distal femur malignant tumor were selected out of the patients that came to our department for treatment of bone tumors from January 2007 to January 2012. The patients were most young people at the average age of 25 within the range of 0-55 years old, including 13 males and 10 females.

\subsection{Methods}

Distal Femur 3D Dissection Model Building and Plate Design. Before operation, patients received X-ray examination and pathological diagnosis to identify the nature of bone tumor, and also received the dual-source 64-slice CT (scanning voltage $120 \mathrm{kV}$ and current $205.59 \mathrm{mAs}$ ) and MRI 3.0T scanning. The profile images of diseased skeleton from scanning were saved in the format of DICOM, and then uploaded into the 3D modelling software Simpleware. The functions including Threshold and Floodfill were used to reconstruct the 3D dissection models for bone tissues and soft tissues at the diseased positions. After that, the CT reconstructed model for hard tissues and the MRI reconstructed model for soft tissues were assembled in the CAD module of the software Simpleware to generate the composite 3D model for bone tumor. The composite model in STL format was uploaded into the reverse engineering software Geomagic to locate the 3D reference plane, design the optimal osteotomy range for the aiding resection plate of tumor resection, and extract the anatomic pattern of femoral model. In the software, a reverse plate was constructed with the same anatomic shape as the front part of femur, and then fitted with the femur to observe the accuracy of resection plate against the femur. After that, the plate was output in STL format.

The STL files of bone tumor model and osteotomy plate from 3D reconstruction were uploaded into the preprocessing software of rapid prototyping equipment, and the laser RP rapid prototyping equipment was utilized to produce the diseased skeleton model and resection plate simultaneously for subsequent use. Before operation, the physical model could help surgeons further understand the conditions of patients, and enable patients and their families to understand the complexity of pathological changes as well as the risk of surgical operation, so as to obtain patients' and their social understanding and cooperation and realize more smooth health education. During operation, assisting tumor resection plate could be installed after exposing the area of bone tumor to guide the osteotomy and realize accurate tumor resection. Moreover, the physical model could, after being disinfected, be compared with the actual object on the operating table to provide reference for accurate resection during surgery.

\section{Results}

Patients should receive periodic follow-up examinations 1 month after operation and every 3 months thereafter, and the X-ray and front and lateral chest radiographs of their affected extremities should be checked. If necessary, CT radiograph might be taken to confirm whether there was any recurrence, prosthesis loosening and pulmonary metastasis. With Enneking scoring system ${ }^{[3]}$, the evaluation of lower extremities covered 6 functions, i.e. pain, ability of motion, patient's acceptance, use of supporting device, ability of walk and gait, 5 points each and 30 points in total. The score of 24-30 points was Excellent, 18-23 points Good, 12-17 points OK and less than 12 points Poor. The evaluation of functions was conducted more than 6 months after operation to find 16 Excellent cases, 5 Good cases and 2 OK cases. The average score was 21 points according to Enneking 
system. Hence, the effect was satisfying.

\section{Discussion}

This paper presents a new approach for surgery, which utilizes computer aided and 3D printing technology to design the individual resection plate for treatment of bone tumor. The individual resection plate can be used not only in the resection of bone tumor, but also to select the optimal reconstruction scheme for excising various allografts, and provide a large amount of clinical data for reference. The technology has been applied in 23 clinical cases.

In the department of bone tumor, the accurate resection of tumor margin and the limb salvage treatment are very important. Accurate preoperative planning and rapid prototyping technology can provide the intraoperative guidance to reduce the duration of operation, lower the amount of intraoperative bleeding, and realize lower occurrence rate of infection complication. Infection is a major complication after operation. Ahlmann et al. ${ }^{[4]}$ reported that $16.6 \%$ of 211 patients with prosthesis replacement for proximal femur, distal femur and proximal tibia tumors experienced the prosthesis failure, while up to $5.2 \%$ of these patients suffered the failure of surgery due to infection. Meanwhile, infection happened 2.5-55.6 months after operation in most cases, and the average time was 6 months. Malawer et al. ${ }^{[5]}$ claimed that the postoperative prosthesis infection rate was 13\%, which was particularly significant to proximal tibia prosthesis. When patients survived for a longer time, the occurrence rate of aseptic loosening and fracture happened to distal prosthesis increased gradually. Unwin et al. ${ }^{[6]}$ arranged the follow-up visits to 218 patients receiving the knee joint salvage treatment with hinge prosthesis in 56 months after operation on average, and found that 5\% experienced prosthesis loosening, 3\% broken stem of prosthesis, and $2 \%$ infection. After their follow-up visits to patients receiving the treatment with prosthesis for lower extremity tumor more than 10 years ago, Mittermayer et al. ${ }^{[7]}$ reported that up 27\% experienced aseptic loosening. When digital technology was utilized to design osteotomy plate, 3D printing technology was employed to assist bone tumor resection, and antibiotics were used for regular postoperative preventive treatment, the 2-year follow-up visits revealed no infection, prosthesis loosening or other complications.

\subsection{Advantages.}

In the CAD and 3D printing technology aided bone tumor resection surgery, CT, MRI and other image fusion technology can be used to identify the margin of bone tumor before operation. The 3D model can help assist the resection of bone tumor and realize less surgical trauma during operation, shorten the duration of operation, lower the amount of interoperative bleeding, and achieve the lower occurrence rate of complications. Meanwhile, prosthesis replacement is often the first choice for postoperative reconstruction to patients with distal femur bone tumor, so the 3D printing technology can ensure no prosthesis loosening after operation for patients, which improves their satisfaction. The operation is simulated to find out the problems that may encounter during operation, so as to prepare the preventive measures in advance. Moreover, it can help find out the advantages and disadvantages of schemes, and determine the optimal surgical scheme. Before operation, repeated practice and communication can be conducted on the computer, so as to get more familiar with the surgical scheme. Before operation, the 3D model can be used to simulate and demonstrate the operation for patients and their families, so as to alleviate their psychological burden and increase the confidence of patients.

The clinical application of computer aided technology in the treatment of pelvic tumors has been reported ${ }^{[8-10]}$, but the $3 \mathrm{D}$ model for pelvis of patient is often reconstructed on the basis of CT scanning, the rapid prototyping technology is used to prepare a physical model of the same size as the pelvis of patient, and then the physical model is used for simulation of operation and design of 
prosthesis.

In this paper, it is believed that the treatment scheme using CAD to design the individual operation aiding plate and simulate the tumor resection and reconstruction of femur during operation can guarantee the accuracy and reliability of femur tumor resection, and bring the surgery of bone tumor into the stage of individual treatment. In this way, the operation will be more accurate, reliable and simple, and the treatment will be more effective.

\subsection{Limitations.}

Along with the application of CAD and 3D printing technologies in the surgery of bone tumor, young surgeons will be much more capable of preoperative simulation and accurate resection of diseased tissues during operation. However, there are still some limitations. If the tumor margin is not accurately reconstructed or the most suitable osteotomy plate is not designed before operation, it will affect the amount of bone to be reduced during operation, and cause inaccurate osteotomy. In this case, it will result in the recurrence of tumor after operation, severely affect the healing of patients and cause great pain to patients. For this reason, preoperative design must be guided and assisted by senior surgeons with rich clinical experience to realize the individual and accurate operation.

\section{Acknowledgments}

This work was supported by The study of c-Myc and mTOR inhibitors in the combined targeting therapy of osteosarcoma. Jilin provincial finance department. (No 3D517P373430)

\section{References}

[1] Wu Hongying, Liang Huimin, Zeng Xiangjie. Application of CT window technology in diagnosis of malignant bone tumors. Journal of Clinical Radiology, 2001, 20 (2): 132-133.

[2]Yu Xiuchun, Liu Xiaoping, Zhou Yin et al. Complementary effect of CT and X-ray examination in diagnosis of bone tumors. Orthopedic Journal of China, 1998, 5(3):265.

[3] Ding Chenglong, Liu Aihua, Wang Guangjun. The CT manifestation of the osteosarcoma. : Journal of China Clinic Medical Imaging, 2000, 11(4):291-292.

[4] Cao Laibin, Xu Aide, Xu Deyong. Practical Bone and Joint Imaging Diagnosis [M]. Ji'nan: Shandong Science and Technology Press, 1998. 326-338.

[5] Azouz EM, Babyn PS, Mascia AT. CT of the abnormal pediat-ric hand wrist with plain film correlation. J Comput AssistT omogr, 1998, 22:252.

[6] Zhou Hai, Lian Zong. Application of medical imaging in the diagnosis of bone tumors in extremities. Foreign Medical Sciences Clinical Radiological Fascicle, 1996, 19(4):205-208.

[7] Hermann G, Klein MJ, Abdelwahab IF, et al. MRI appear-ance of primary non-hodgkins lymphoma of bone. Skeletal Radiol, 1997, 26(11):629-632.

[8] Pepys MB. Pathogenesis, diagnosis and treatment of sy stemicamyloidosis. Philos Trans R Soc Lond Biol Sci, 2001, 356:203-210.

[9] Liu Gang, Zou Wanzhong. Early diagnosis of renal amyloidosis. Chinese Journal of Internal Medicine, 2005, 44(3):234.

[10] Falk RH, Comenzo RL, Skinner M. The systemic amyloid-oses . N Engl J Med, 1997, 337:898-909. 